\title{
Short note: Least fixed points versus least closed points
}

\author{
Gerhard Jäger ${ }^{1}$ (1)
}

Received: 12 March 2020 / Accepted: 22 January 2021 / Published online: 8 February 2021

(c) The Author(s) 2021

\section{Abstract}

This short note is on the question whether the intersection of all fixed points of a positive arithmetic operator and the intersection of all its closed points can proved to be equivalent in a weak fragment of second order arithmetic.

Keywords Second order arithmetic · Fixed points - Least fixed points and least closed points of $X$-positive operator forms

Mathematics Subject Classification 03F25 · 03F35

\section{Introduction}

Fixed points and least fixed points of positive operators are well-studied objects; cf., e.g., Moschovakis [1] for the general theory and Pohlers [2] for applications in proof theory. Given a monotone operator

$$
\Gamma: \operatorname{Pow}(\mathbb{N}) \rightarrow \operatorname{Pow}(\mathbb{N}),
$$

the Knaster-Tarski theorem tells us that $\Gamma$ has a least fixed point $I_{\Gamma}$ and that

$$
I_{\Gamma}=\bigcap\{X \subseteq \mathbb{N}: \Gamma(X)=X\}=\bigcap\{X \subseteq \mathbb{N}: \Gamma(X) \subseteq X\} .
$$

Thus the least fixed point of $\Gamma$ can be characterized as the intersection of all fixed points of $\Gamma$ as well as the intersection of all $\Gamma$-closed points.

However, according to my knowledge, it has never been discussed whether this equivalence can be proved in weak subsystems of second order arithmetic. It is the purpose of this note to shed some light on this issue. I thank Kentaro Sato for bringing this question to my attention.

Gerhard Jäger

gerhard.jaeger@inf.unibe.ch

1 Institut für Informatik, Universität Bern, Neubrückstrasse 10, 3012 Bern, Switzerland 


\section{The general environment}

Let $\mathcal{L}_{2}$ be a standard language of second order arithmetic with countably infinite supplies of number variables $k, m, n$ and set variables $U, V, X, Y, Z$. We also have function and relation symbols for all primitive recursive functions and relations. The formulas $\theta, \varphi, \psi$ of $\mathcal{L}_{2}$ are built up as usual. See, for example, Simpson [3].

A formula of $\mathcal{L}_{2}$ without bound set variables is called arithmetical. We refer to arithmetical formulas $\varphi[X, n]$ in which the set variable $X$ occurs only positively as $X$-positive operator forms. Each such $X$-positive operator form $\varphi[X, n]$ defines a monotone function

$$
\Gamma_{\varphi}: \operatorname{Pow}(\mathbb{N}) \rightarrow \operatorname{Pow}(\mathbb{N}) ; \quad \Gamma_{\varphi}(S):=\{n \in \mathbb{N}: \varphi[S, n]\}
$$

Throughout this paper we work in classical logic with equality for the first sort. Equality for sets in $\mathcal{L}_{2}$ is defined by saying that two sets are identical iff they contain the same elements.

$\mathrm{ACA}_{0}$ is the system of second order arithmetic whose non-logical axioms comprise the defining axioms for all primitive recursive functions and relations, the axiom schema of arithmetical comprehension

$$
\exists X \forall n(n \in X \leftrightarrow \varphi[n])
$$

for all arithmetic formulas $\varphi[n]$, and the induction axiom

$$
\forall X(0 \in X \wedge \forall n(n \in X \rightarrow n+1 \in X) \rightarrow \forall n(n \in X))
$$

It is well known that $A C A_{0}$ is a conservative extension of Peano arithmetic PA.

Now we turn to the notions of fixed point and least fixed point as well as closed point and least closed point of $X$-positive operator forms. Let $\varphi[X, n]$ be such an operator form; then we set:

$$
\begin{aligned}
F P_{\varphi}[Z] & :=\forall n(\varphi[Z, n] \leftrightarrow n \in Z), \\
L F P_{\varphi}[Z] & :=F P_{\varphi}[Z] \wedge \forall X\left(F P_{\varphi}[X] \rightarrow Z \subseteq X\right), \\
C P_{\varphi}[Z] & :=\forall n(\varphi[Z, n] \rightarrow n \in Z), \\
L C P_{\varphi}[Z] & :=C P_{\varphi}[Z] \wedge \forall X\left(C P_{\varphi}[X] \rightarrow Z \subseteq X\right) .
\end{aligned}
$$

Before we turn to some systems of second order arithmetic based on these principles, let me point out a significant difference between fixed points and closed points.

Obviously, every fixed point of an $X$-positive operator form is also a closed point of this form. The converse is clearly not true in general. More interesting is the observation that closed points are closed under intersections whereas this is in general not the case for fixed points. 
Remark 1 Clearly, the closed points of any $X$-positive operator form $\varphi[X, n]$ form are closed under intersections, i.e.

$$
\mathrm{ACA}_{0} \vdash \forall X, Y\left(C P_{\varphi}[X] \wedge C P_{\varphi}[Y] \rightarrow C P_{\varphi}[X \cap Y]\right)
$$

On the other hand, consider the $X$-positive operator form

$$
\psi[X, n]:=(\exists m \in X)((n=m \wedge m<2) \vee n=m+2 \vee n=m+5) .
$$

Then it is easy to check that

$$
P_{\psi}:=\{2 m+5 n: m, n \in \mathbb{N}\} \text { and } Q_{\psi}:=\{1+2 m+5 n: m, n \in \mathbb{N}\}
$$

are fixed points of $\psi$ whereas $P_{\psi} \cap Q_{\psi}$ is not a fixed point of $\psi$.

In the following we write $\mathrm{FP}_{0}$, $\mathrm{LFP}_{0}$, and $\mathrm{LCP}_{0}$ for the extensions of $A C A_{0}$ by all formulas

$$
\exists Z F P_{\varphi}[Z], \quad \exists Z L F P_{\varphi}[Z] \text {, and } \exists Z L C P_{\varphi}[Z] \text {, }
$$

respectively, where $\varphi[X, n]$ ranges over all $X$-positive operator forms. Keep in mind that $\varphi[X, n]$ may also contain parameters, i.e., additional free number and set variables.

\section{$3 L F P$ versus $L C P$}

We first observe (trivial) that least fixed points and least closed points of $X$-positive operator forms are uniquely determined, provided that they can be shown to exist. It is also easy to see that the least closed point of any $X$-positive operator form is its least fixed point. And, as the following lemma states, this fact can be proved in $A C A_{0}$.

Lemma 2 Let $\varphi[X, n]$ be an $X$-positive operator form. Then we have that

$$
\mathrm{ACA}_{0} \vdash \forall Z\left(L C P_{\varphi}[Z] \rightarrow \operatorname{LFP}_{\varphi}[Z]\right)
$$

Proof Let $Z$ be the least closed point of $\varphi$. We form, by means of arithmetic comprehension, the set

$$
Y:=\{n: \varphi[Z, n]\}
$$

Because of $C P_{\varphi}[Z]$ we have $Y \subseteq Z$, and thus the monotonicity of $\varphi$ yields

$$
\forall n(\varphi[Y, n] \rightarrow n \in Y)
$$

So we have $C P_{\varphi}[Y]$, hence $Z \subseteq Y$. Therefore, $Z$ is a fixed point of $\varphi$. On the other hand, every fixed point of $\varphi$ is also $\varphi$-closed. Hence $Z$ is the least fixed point of $\varphi$. 
More interesting is the question of whether the least fixed point of an $X$-positive operator is its least closed point. In order to obtain this result, we have to make use of a specific fixed point construction and thus work in $\mathrm{FP}_{0}$.

Theorem 3 Let $\varphi[X, n]$ be an $X$-positive operator form. Then we have that

$$
\mathrm{FP}_{0} \vdash \forall Z\left(L F P_{\varphi}[Z] \rightarrow \operatorname{LCP}_{\varphi}[Z]\right)
$$

Proof Working in $\mathrm{FP}_{0}$ we assume that $Z$ is the least fixed point of $\varphi$. We must show that

$$
C P_{\varphi}[Y] \rightarrow Z \subseteq Y
$$

for any set $Y$. Given a $Y$ with $C P_{\varphi}[Y]$, consider the $X$-positive operator form

$$
\psi[X, n]:=\varphi[X, n] \wedge n \in Y
$$

Since we work in $\mathrm{FP}_{0}$, we know that $\psi$ has a fixed point $U$, i.e.

$$
\forall n(n \in U \leftrightarrow \psi[U, n] \leftrightarrow \varphi[U, n] \wedge n \in Y),
$$

in particular, $U \subseteq Y$. Therefore, for any $n$,

$$
\varphi[U, n] \rightarrow \varphi[Y, n] \rightarrow n \in Y
$$

Hence,

$$
\varphi[U, n] \leftrightarrow \varphi[U, n] \wedge n \in Y \leftrightarrow \psi[U, n] \leftrightarrow n \in U
$$

This means that $U$ is a fixed point of $\varphi$, and we obtain

$$
Z \subseteq U \subseteq Y
$$

Thus we have $(*)$, and our theorem is proved.

Corollary 4 The theories $\mathrm{LFP}_{0}$ and $\mathrm{LCP}_{0}$ are equivalent, i.e., for every formula $\theta$ of $\mathcal{L}_{2}$,

$$
\mathrm{LFP}_{0} \vdash \theta \quad \Longleftrightarrow \quad \mathrm{LCP}_{0} \vdash \theta
$$

Proof For the direction from left to right we only have to show that $\mathrm{LCP}_{0}$ proves $\exists Z L F P \varphi[Z]$ for any $X$-positive operator form $\varphi[X, n]$. This is immediate from the previous lemma.

Conversely, for the direction from right to left, all we have to do is to prove that $\mathrm{LFP}_{0}$ proves $\exists Z L C P_{\varphi}[Z]$ for any $X$-positive operator form $\varphi[X, n]$. But since $\mathrm{FP}_{0}$ is a subsystem of $L F P_{0}$, this is a direct consequence of the previous theorem. 
The equivalence of $\mathrm{LFP}_{0}$ and $\mathrm{LCP}_{0}$ can also be shown more directly: $\mathrm{LFP}_{0} \subseteq \mathrm{LCP}_{0}$ is obvious (see above). For the inclusion $\mathrm{LCP}_{0} \subseteq \mathrm{LFP}_{0}$, pick an arbitrary $X$-positive operator form $\varphi[X, n]$. We have to show in $\operatorname{LFP}_{0}$ that $\varphi$ has a least closed point. To achieve this, consider - following a suggestion of Sato - the $X$-positive operator form

$$
\psi[X, n]:=\varphi[X, n] \vee n \in X
$$

Then it is easy to check that

$$
\mathrm{ACA}_{0} \vdash \forall Z\left(F P_{\psi}[Z] \leftrightarrow C P_{\varphi}[Z]\right)
$$

As a consequence of that we know that the least fixed point of $\psi$ is the least closed point of $\varphi$. This argument shows $\mathrm{LCP}_{0} \subseteq \mathrm{LFP}_{0}$.

There is an interesting questions in this context, which is still open as far as I know: Can the assertion $\forall Z\left(\operatorname{LFP}_{\varphi}[Z] \rightarrow L C P_{\varphi}[Z]\right)$ be proved in $A_{C A}$ ? And if not, what do we need? Is there a theory weaker than $\mathrm{FP}_{0}$ that does the job?

Funding Open Access funding provided by Universität Bern

Open Access This article is licensed under a Creative Commons Attribution 4.0 International License, which permits use, sharing, adaptation, distribution and reproduction in any medium or format, as long as you give appropriate credit to the original author(s) and the source, provide a link to the Creative Commons licence, and indicate if changes were made. The images or other third party material in this article are included in the article's Creative Commons licence, unless indicated otherwise in a credit line to the material. If material is not included in the article's Creative Commons licence and your intended use is not permitted by statutory regulation or exceeds the permitted use, you will need to obtain permission directly from the copyright holder. To view a copy of this licence, visit http://creativecommons.org/licenses/by/4.0/.

\section{References}

1. Moschovakis, J.N.: Elementary Induction on Abstract Structures. Studies in Logic and the Foundations of Mathematics, no. 77. North-Holland, Amsterdam (1974)

2. Pohlers, W.: Proof Theory, the First Step into Impredicativity. Universitext. Springer, Berlin (2010)

3. Simpson, S.G.: Subsystems of Second Order Arithmetic. Perspectives in Logic, 2d edn. Association for Symbolic Logic and Cambridge University Press, Cambridge (2009)

Publisher's Note Springer Nature remains neutral with regard to jurisdictional claims in published maps and institutional affiliations. 